\title{
Comment on Trujillo-Tiebas MJ et al. J Assist Reprod Genet DOI 10.1007/s10815-009-9339-1
}

\author{
Dong-Zhi Li
}

Received: 5 October 2009 /Accepted: 23 November 2009 /Published online: 29 December 2009

(C) Springer Science+Business Media, LLC 2009

I read with interest the recent article by Trujillo-Tiebas et al. [1], which reported prenatal diagnosis of skeletal dysplasia due to FGFR3 gene mutations at a Spanish center during a 9 -year period. They confirmed two cases of achondroplasia and four cases of thanatophoric dysplasia in 34 prenatal samples using DNA analysis methods, and concluded that molecular studies in ongoing pregnancies are indicated in cases with an affected parent, an abnormal sonographic finding corresponding to the indicated disorders, or a family history with positive molecular studies. However, in an obstetrician's experience, the conclusion of this study might not be so convincing.

First, the indication in some cases for an invasive testing was not with careful consideration, at least to some extent. For example, in the $19 \mathrm{CVS}$ cases, 10 samplings were done just for maternal anxiety. The results were, not surprisingly, that only 2 CVS samples were positive and both because of a maternal achondroplasia. Indeed, these abortion riskrelated procedures could have been avoided in most cases. The recurrent risk is very rare for a sporadic skeletal dysplasia with FGFR3 gene mutations. The maternal anxiety can be alleviated effectively with genetic counseling providing accurate information about the autosomal dominant hereditary conditions.

Second, what was the reason that amniocentesis had been done in a near term pregnancy? There were 15 amniotic fluid samples at $13-35$ weeks. The authors said

\section{D.-Z. Li $(\bowtie)$}

Prenatal Diagnostic Center, Guangzhou Maternal \& Neonatal Hospital, Guangzhou Medical College,

Renminzhong Road 402,

Guangzhou, Guangdong 510180, People's Republic of China

e-mail: dongzhi3@yahoo.com.cn that the legal termination of pregnancy is not permitted beyond 22 weeks in Spain, so what the purpose was for those pregnancies in which an amniocentesis was given at a third trimester? In the 15 amniotic fluid samples, there were 4 cases with a positive molecular test, all of which had a hypoplastic thorax plus micromelia. What were the pregnancy outcomes for these 4 thanatophoric dysplasia cases and those with a negative molecular test?

Third, what method was used to rule out chromosomal abnormalities in those samples from abortuses and from amniotic fluid sampled at a late gestation? The authors described a multiplex QF-PCR assay for common aneuploidies detection. Surprisingly, they included four sets of STR markers for chromosomes $13,15,18$, and $\mathrm{X}$ rather than a combination of chromosomes 21 .

Finally, twenty abortus samples were also tested in this study, and indications were skeletal dysplasia in 16 cases, thanatophoric dysplasia in 2 cases, and achondroplasia in the remaining 2 cases. Although not mentioned by the authors, the pregnancies involved might have been terminated because of severe ultrasound abnormalities. Why did these fetuses have not been sampled prenatally suggested by this study instead of direct aborting?

The skeletal dysplasias are a large and heterogeneous group of disorders. Currently, there are more than 100 recognized forms of skeletal dysplasia, which makes arriving at a specific diagnosis difficult [2]. The prevalence of skeletal dysplasias (excluding limb amputations) is estimated at 2.4 per 10,000 births [3]. Many manifest in the prenatal period, making them amenable to ultrasound prenatal diagnosis. However, to make a definite diagnosis, complementary radiological, pathological and molecular studies after birth are mandatory. Only once a limited differential diagnosis has been established, should molecular investigations be considered in utero. 


\section{References}

1. Trujillo-Tiebas MJ, Fenollar-Cortés M, Lorda-Sánchez I, DíazRecasens J, Carrillo Redondo A, Ramos-Corrales C, et al. Prenatal diagnosis of skeletal dysplasia due to FGFR3 gene mutations: a 9-year experience : Prenatal diagnosis in FGFR3 gene. J Assist Reprod Genet. 2009;26:455-60.
2. Dighe M, Fligner C, Cheng E, Warren B, Dubinsky T. Fetal skeletal dysplasia: an approach to diagnosis with illustrative cases. Radiographics. 2008;28:1061-77.

3. Rasmussen SA, Bieber FR, Benecerraf BR, Lachman RS, Rimoin DL, Holmes LB. Epidemiology of osteochondrodysplasias: changing trends due to advances in prenatal diagnosis. Am J Med Genet. 1996;61:49-58. 